Interplay of host volume variations and internal distortions in the course of intercalation into disordered matrices

E. V. VakarinGermà Garcia-BelmonteJ. P. Badiali

Citation: The Journal of Chemical Physics 126, 234709 (2007); doi: 10.1063/1.2743410

View online: http://dx.doi.org/10.1063/1.2743410

View Table of Contents: http://aip.scitation.org/toc/jcp/126/23

Published by the American Institute of Physics

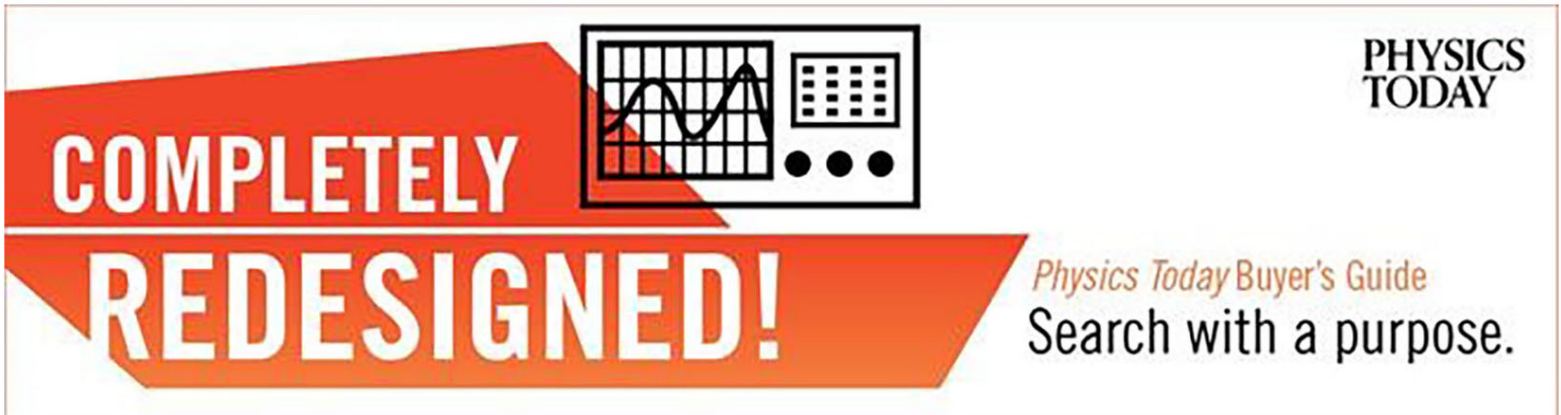




\title{
Interplay of host volume variations and internal distortions in the course of intercalation into disordered matrices
}

\author{
E. V. Vakarin \\ UMR 7575 LECA ENSCP-UPMC-CNRS, 11 rue P. et M. Curie, 75231 Cedex 05 Paris, France \\ Germà Garcia-Belmonte \\ Departament de Física, Universitat Jaume I, E-12071 Castelló, Spain \\ J. P. Badiali \\ UMR 7575 LECA ENSCP-UPMC-CNRS, 11 rue P. et M. Curie, 75231 Cedex 05 Paris, France
}

(Received 26 February 2007; accepted 25 April 2007; published online 20 June 2007)

\begin{abstract}
Based on a combination of the distortive lattice gas model and the maximum information entropy approach, the thermodynamics of insertion into disordered hosts is analyzed. It is found that the isotherm specificities can be explained as a cooperative interplay of the host volume expansion and the internal distortions, which tend to optimize the host structure inducing a local lowering of the insertion energetic cost. Behavior of amorphous $\mathrm{Li}_{x} \mathrm{WO}_{3}$ films of different thicknesses is discussed in this context. (c) 2007 American Institute of Physics. [DOI: 10.1063/1.2743410]
\end{abstract}

\section{INTRODUCTION}

Insertion processes have received considerable interest because of their applications in a variety of technologically important domains, e.g., storage devices, ${ }^{1,2}$ high capacity batteries, electrochromic devices, solar cells, etc. (see Ref. 3 for a review). These materials are usually produced by insertion or doping of a host matrix by neutral or charged guest species. Typical examples are the intercalation compounds or conducting polymers. In many cases (e.g., amorphous/ porous materials or polymer films) the host matrix is spatially disordered. This has some advantages ${ }^{4}$ (e.g., higher capacity) in comparison to crystalline hosts. In particular, this allows one to avoid the macroscopic insertion-induced phase transformations, such as restructuring (crystalline $\mathrm{WO}_{3}$ or staging (graphite). In its turn, this broadens the voltage and composition ranges where a stable cycling can be maintained.

From a theoretical point of view the host disorder is usually described by a distribution of some relevant quantity, such as pore sizes or site energies. ${ }^{5-7}$ Then the insertion isotherm is represented as an average over the host fluctuations. Despite a considerable progress ${ }^{5}$ in the field, the consequences of the disordered host morphology are still poorly understood. In particular, recent theoretical studies ${ }^{8,9}$ of adsorption into disordered porous media suggest that, because of the host disorder, the adsorption thermodynamics might depend on a driving path (e.g., controlled injections of prescribed portions or an equilibrium with a bulk reservoir). In the context of intercalation processes this means that different electrochemical methods (e.g., chronopotentiometry and voltammetry) could give different results. Therefore, analyzing a system by different techniques could help in the characterization of the host morphology.

The problem is complicated by the structural changes occurring upon insertion ${ }^{10,11}$ or intercalation. ${ }^{12,13}$ In many cases mechanical strain generated in the intercalation/ deintercalation cycles induces structural as well as volume changes in the electrode material, leading to fracturing, cracking and even crumbling, and thereby to electrode irreversibility upon cycling. One of the most challenging issues in the development of devices based on lithium intercalation materials is the control over structural changes and deformations produced by lithium insertion/removal. These can be manifested as spatially distributed internal distortions which lead either to topotactic insertion ${ }^{4}$ or even to macroscopic (up to $10 \%$ ) volume dilatations. ${ }^{13,14}$ The latter are easily detectable (e.g., by optical profilometry ${ }^{13}$ ), while the internal distortions are rather difficult to control. For this reason quite often the internal host structure remains poorly characterized. On the other hand, a coupling between the insertion and the dilatation modes has been shown ${ }^{15}$ to be responsible for well-pronounced thermodynamic features. Even in the absence of detectable volume variations, the internal host distortion should contribute ${ }^{16}$ a fluctuational term to the insertion thermodynamics. Therefore, one should be able (at least in principle) to distinguish these two effects.

In the absence of a detailed microscopic information one has to analyze the underlying physics directly from the experimental data and a plausible insertion model. The aim of this work is to develop a holistic theoretical scheme, able to extract from an experimental isotherm $\mu_{b}(x)$ the volume variation and the internal distortion traces. This would allow us to analyze their impacts to the observed behavior. With this purpose we combine the distortive lattice gas model that has been successfully applied ${ }^{17}$ to a description of volume dilatation in ordered intercalation compounds with the maximum information entropy approach. ${ }^{18}$ The latter is an inductive inference method which is quite efficient ${ }^{9}$ in estimating the host disorder from the experimental isotherm data. Then we test our predictions, trying to explain some puzzling points in the recent experimental results. ${ }^{13}$ 


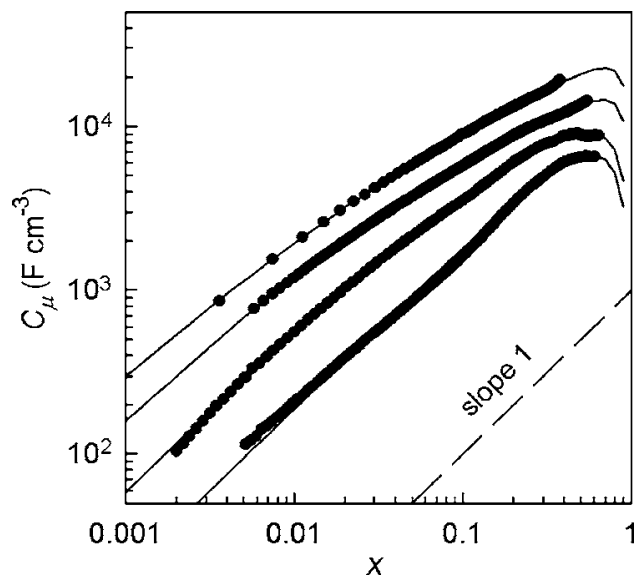

FIG. 1. Equilibrium film capacitance $C_{\mu} \propto \partial x / \partial \mu$ as a function of the composition (molar fraction) $x$ for film thickness (in nm) of 100, 200, 300, and 400 (top). Solid lines correspond to fits using Eq. (1). To improve the clarity of the graph the capacitance corresponding to 200,300 , and $400 \mathrm{~nm}$ is multiplied by 2, 4, and 8, respectively. A reference dashed line of slope 1 is also shown. Reprinted with permission from Journal of Physical Chemistry B, v. 110, p. 4514 (2006). Copyright (2006) American Chemical Society.

\section{OVERVIEW OF EXPERIMENTAL RESULTS}

Recent experiments ${ }^{13,14}$ on amorphous $\mathrm{Li}_{x} \mathrm{WO}_{3}$ compounds reveal that the insertion thermodynamics differs remarkably from the lattice gas picture, conventionally used for the description of intercalation into rigid crystalline matrices. In particular, the isotherms and the capacitance curves have been shown to involve power-law dependencies on the guest concentration $x$ (Ref. 13),

$$
\mu_{b}=E_{0}+(1+\eta) G x^{\eta}+\frac{1}{\beta} \ln \left(\frac{x}{1-x}\right) .
$$

The capacitance $C_{\mu} \propto\left[\partial \mu_{b} / \partial x\right]^{-1}$ that follows from Eq. (1), has been found in an excellent agreement with the experimental data. This is demonstrated in Fig. 1 for the case of lithium intercalation into amorphous $\mathrm{WO}_{3}$ films of different thicknesses. Therefore, in what follows this relation [Eq. (1)] will be considered as an experimental fact. Here $G$ was interpreted as a host-guest interaction parameter. However, it has been found to depend on the host film thickness. For the thinnest $(100 \mathrm{~nm})$ film the intercalant-host interaction term $G$ is not detectable $(G \approx 0)$ so that the system behaves like an ideal noninteracting lattice gas in which entropic contributions play the determining role. As the thickness increases $G$ becomes larger, attaining values around $350 \mathrm{meV}$ for thicker $(400 \mathrm{~nm})$ films, what modifies the asymptotic behavior of the chemical capacitance (see Fig. 1). The exponent of the intercalant-host interaction term has been found to be around $\eta \approx 0.5$. Such a variation of the host-guest interaction parameter is difficult to understand because the interaction between the two subsystems is essentially determined by their chemical nature. Variation of $G$ with the thickness are accompanied by specific volume changes of the host matrix. ${ }^{13}$ The relative volume changes at $x=0.4$ of a $200 \mathrm{~nm}$ amorphous $\mathrm{Li}_{x} \mathrm{WO}_{3}$ film are shown in Fig. 2, as a topographic representation of the height reached by the outer surface of the film. Volume expansion is observed to yield a highly rough surface in the intercalated part. All films showed relative volume changes near $10 \%$ on average, except for the 100-nm-thick samples which presented no expansion at all, in agreement with the results of electrochemical measurements $(G \approx 0)$.

Therefore, the experiments ${ }^{13,14}$ (Figs. 1 and 2) provide an evidence for the significant role played by the film volume change and the surface roughness in the underlying intercalation thermodynamics (cell voltage variation with the guest composition). Importantly, these results make apparent the occurrence of some sort of thickness effects which are poorly understood.

\section{VOLUME VARIATIONS}

The host volume variations can be taken into account in the framework of the lattice gas model combined with the continuum elasticity theory. This leads to the following isotherm: ${ }^{17}$

$$
\mu=\mu_{0}(x)+S(x) \frac{d \epsilon(x)}{d x}-\frac{d \sigma(x)}{d x} \epsilon(x),
$$

where $\beta \mu_{0}(x)=\beta E_{0}+\ln (x /(1-x))$ is the standard lattice gas part without the guest interaction, $S(x)=\Lambda \epsilon(x)-\sigma(x)$ is the

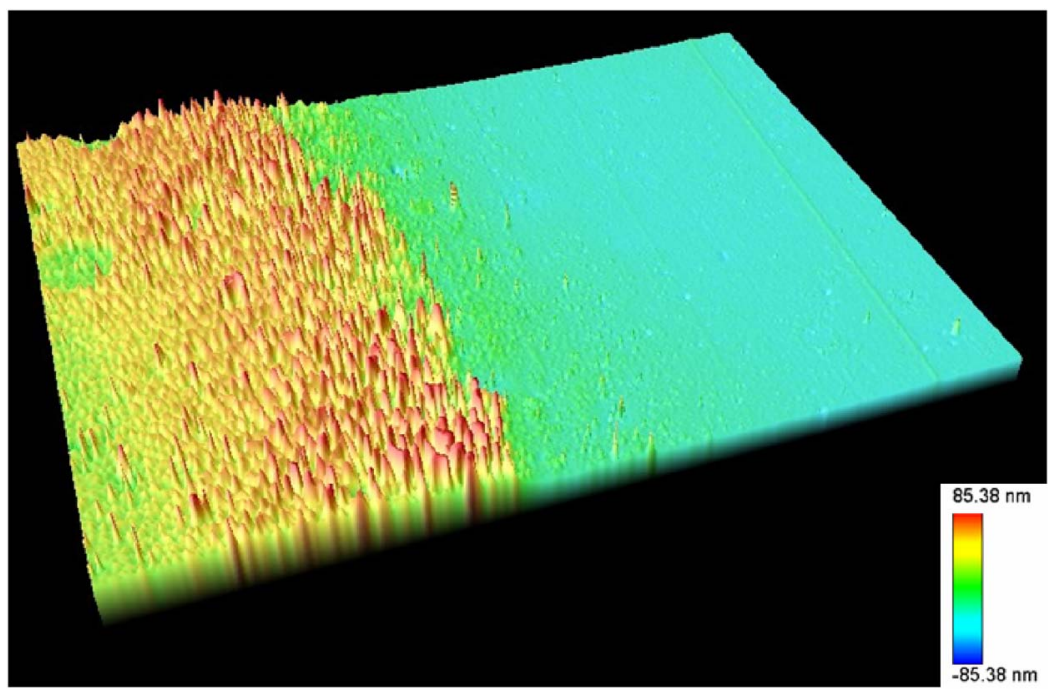

FIG. 2. Topography of a 200-nm-thick amorphous $\mathrm{Li}_{x} \mathrm{WO}_{3}$ film at $x=0.4$. The border between the intercalated (left part of the image) and the nonintercalated (right part of the image) fields is clearly seen. The average volume change is about $10 \%$. Scanned area is $637 \times 477 \mu \mathrm{m}^{2}$. Reprinted with permission from Journal of Physical Chemistry B, v. 110, p. 4514 (2006). Copyright (2006) American Chemical Society. 
concentration dependent total stress. The latter involves the external (loading) stress $\sigma(x)$ and the internal stress $\Lambda \epsilon(x)$. Here $\Lambda$ is the elastic constant related to the Young modulus and $\epsilon(x)$ is the strain which results from the changes in the host volume $V(x)$. In the case of unclamped matrices we may neglect the loading contribution, arriving at

$$
\mu=\mu_{0}(x)+\Lambda \delta^{2} p(x) \frac{d p(x)}{d x},
$$

where $\delta=[V(1)-V(0)] / V(0)$ is the relative volume variation and $p(x)=[V(x)-V(0)] /[V(1)-V(0)]$ is the modulating function with $p(0)=0$ and $p(1)=1$. Identifying the elastic part in Eq. (3) with the power-law term in Eq. (1) and solving with respect to $p(x)$ one gets a sublinear (recall that $\eta \approx 0.5$ ) modulating function ${ }^{13}$

$$
p(x)=x^{(1+\eta) / 2}
$$

and the coupling of the empirical parameter $G$ and the relative volume expansion

$$
\delta=\left(\frac{G}{\Lambda}\right)^{1 / 2}
$$

It has been shown ${ }^{13}$ that the capacitance derived from Eq. (3) predicts the film volume expansion around $10 \%$ (on average) at the intercalation level $x \approx 0.4$. These volume changes have been directly observed by optical profilometry for the film thickness $\geqslant 200 \mathrm{~nm}$ (Fig. 2). Nevertheless, these results do not allow to account for the thresholdlike behavior ${ }^{13}$ of the volume expansion with the film thickness.

\section{INTERNAL DISTORTIONS}

The experimental results ${ }^{13}$ clearly indicate that the matrix volume variation is accompanied by an extensive roughening of the surface in the intercalated region. Based on this we suppose that in addition to the dilatation the host undergoes some internal distortions. These are related to an adjustment of the host structure allowing for a more efficient accommodation. This can be taken into account as a correction $\varphi$ to the host-guest interaction, changing the insertion energetic cost

$$
\mu(x \mid \varphi)=\varphi+\mu_{0}(x)+\Lambda \delta^{2} p(x) \frac{d p(x)}{d x},
$$

where $\varphi$ is a fluctuating quantity distributed according to some probability density $f(\varphi)$. The latter is unknown, the only available experimental evidence we have is the isotherm (1). Therefore, we infer the distribution using the maximum information entropy approach., ${ }^{9,19}$ The procedure consists in maximizing the Shannon entropy measure

$$
H=-\int d \varphi f(\varphi) \ln f(\varphi)
$$

under the constraint that the average $\overline{\mu(\varphi)}$ reproduces the experimental data (1)

$$
\mu_{b}=\overline{\mu(x \mid \varphi)}=\int d \varphi f(\varphi) \mu(x \mid \varphi) .
$$

This gives the following concentration dependent distribution:

$$
f(\varphi \mid x)=\frac{\exp [\lambda(x) \mu(x \mid \varphi)]}{\int d \varphi \exp [\lambda(x) \mu(x \mid \varphi)]}
$$

where the Lagrange multiplier $\lambda(x)$ should be found from the constraint (8). This leads to

$$
(1+\eta) G x^{\eta}=\varphi(x)+\Lambda \delta^{2} p(x) \frac{d p(x)}{d x},
$$

where $1 / \lambda(x)=\varphi(x)=\bar{\varphi}$. It is seen that the power-law term involves a combination of the distortion and the volume dilatation. These effects have been found to depend on the host film thickness. ${ }^{13}$ Thus it is not surprising that the parameter $G$ is also thickness dependent. On the other hand, Eq. (10) determines the dilatation and the internal distortion impacts to the observed thermodynamics. Having a plausible estimation for one of these ingredients allows us to estimate the other.

\section{ANALYSIS OF EXPERIMENTAL DATA}

In what follows we will try to see to what extent an interplay of the host distortion and dilatation is able to explain the behavior observed in amorphous $\mathrm{Li}_{x} \mathrm{WO}_{3}$ films.

Note that the last term in Eq. (10) includes not only the dilatation magnitude $\delta p(x)$ but also the dilatation rate $R(x)$ $=d p(x) / d x$. Even if (at some point $x=x_{0}$ ) the distortion contributions $\varphi_{1}\left(x_{0}\right), \varphi_{2}\left(x_{0}\right)$ and the volume dilatations $p_{1}\left(x_{0}\right)$, $p_{2}\left(x_{0}\right)$ are the same for two films of different thicknesses, their isotherms could be distinguished by different rates $R_{1}\left(x_{0}\right) \neq R_{2}\left(x_{0}\right)$. This gives an idea on why the host films with almost the same dilatation magnitudes (as in the case of 300 and $400 \mathrm{~nm}$ films at $x_{0}=0.4$ ) exhibit different electrochemical responses. Therefore, measuring simultaneously the isotherm and the concentration dependent volume variations one can get some information on the internal distortions.

On the other hand, having an estimation of the internal host morphology, one can predict the volume variation. In particular, the recent analysis ${ }^{14}$ of the jump diffusion coefficient reveals a power-law dependence of the hopping rate on the concentration. Based on this we may suppose $\varphi(x)$ $=-K x^{q}$, with $K$ being an effective distortion coefficient. Then Eq. (10) can be solved with respect to $p(x)$. This leads to

$$
p(x)=\left[\frac{G}{\Lambda \delta^{2}} x^{\eta+1}+\frac{K}{\Lambda \delta^{2}(q+1)} x^{q+1}\right]^{1 / 2} .
$$

In order to satisfy the condition $p(1)=1$, the following relation should hold: 


$$
\frac{G}{\Lambda \delta^{2}}+\frac{K}{\Lambda \delta^{2}(q+1)}=1
$$

This allows us to estimate the relative host dilatation at $x$ $=1$,

$$
\delta=\left(\frac{G}{\Lambda}+\frac{K}{\Lambda(q+1)}\right)^{1 / 2} .
$$

We thus see that even for $G \approx 0$ (as in the case of $100 \mathrm{~nm}$ films) the dilatation does not vanish because of the distortion contribution. From Eq. (12) one can see that $G=0$ corresponds to a subtle relation $K=\Lambda \delta^{2}(q+1)$ between the dilatation and the internal distortion. The latter is probably rather small for thin films, as this follows from the relatively low surface roughness in comparison to that for the thicker films. ${ }^{13}$ For this reason, in agreement with the experimental observations, the volume expansion could be undetectable. On the other hand, the host has been found to undergo irreversible transformations at $x>0.6$. For this reason the experimental magnitude of $\delta$ is not available. The only information at hand is the volume dilatation $\delta \cdot p(x=0.4)$.

Therefore, we have to analyze the volume variation as a function of the concentration. Combining Eqs. (11) and (12) we arrive at

$$
p(x)=\left[(1-B) x^{\eta+1}+B x^{q+1}\right]^{1 / 2},
$$

where $B=K /\left[\Lambda \delta^{2}(q+1)\right]$ is a parameter measuring the relative impact of the distortion and the volume variation effects. Using Eq. (12) we can relate $B$ to the empirical constant $G$, $B=1-G /\left(\Lambda \delta^{2}\right)$. This suggests that $B<1$ and, depending on the other parameters, can even be negative. This, in its turn, means that the distortion coefficient $K$ might change its sign with increasing film thickness (as the parameter $G$ grows). Taking into account that the isotherm (1) is now represented as

$$
\mu(x)=\mu_{0}(x)-K x^{q}+\Lambda \delta^{2} p(x) \frac{d p(x)}{d x},
$$

we may expect a crossover between two regimes with decreasing host film thickness. For thick films (positive $K$ or $B$ ) the internal distortions favor an adjustment of the host geometry, making the insertion less energetically consuming. For thin films (negative $K$ or $B$ ) we have to spend energy in order to induce both distortions and volume dilatation (presumably because of a stronger influence of the film support). Interestingly that a quite similar crossover from a quenched to an adsorbate-induced roughness has recently been reported ${ }^{20}$ for HCL adsorption on ice films of varying thickness. In the context of the present work the crossover is due to an interplay of the film volume dilatation and the insertion-induced internal distortions. This behavior is illustrated in Fig. 3, where $p(x)$, given by Eq. (14), is plotted for different values of $B$. In order to recover the thresholdlike variation ${ }^{13}$ of the volume with the film thickness we have to assume that the parameter $B$ changes sign with decreasing thickness. However, this makes the volume variation ill defined $\left(p(x)^{2}<0\right)$ in the range of low concentrations. This suggests that for thin films another distortion mechanism is probably operating in this domain. Note that the isotherm remains mathematically

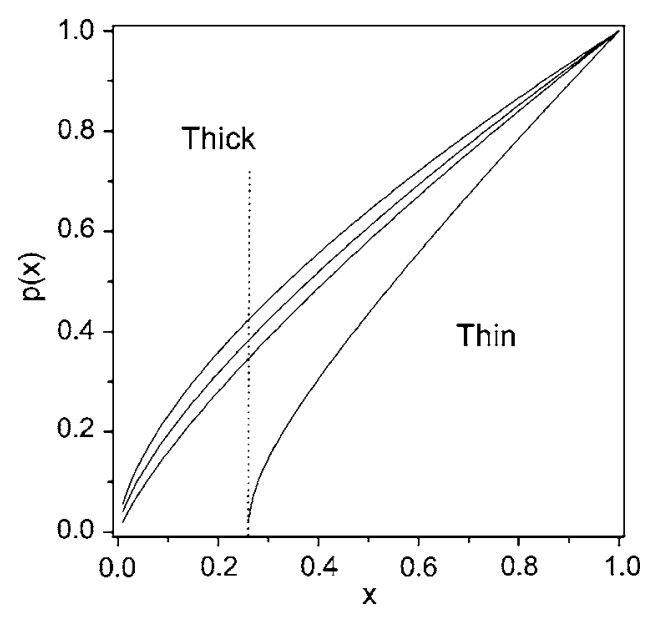

FIG. 3. Modulating function calculated from Eq. (14) at $q=0.2, \eta=0.5$, and different $B=0.7,0.2,-0.2,-2$ (from left to right).

correct because all our estimations are made under the condition (10) preserving the isotherm shape.

On the other hand, the above artifact may result from the concentration dependence accepted for $\varphi(x)$. In other words, the power-law shape of the distortion part cannot completely explain the observed electrochemical response. Therefore, making plausible assumptions on $p(x)$ and solving Eq. (10) with respect to $\varphi(x)$ seems to be more constructive. In the previous study ${ }^{17}$ the following modulating function has been considered:

$$
p(x)=\frac{1}{2}\left[1+\tanh \left(\Delta\left[x-x_{0}\right]\right)\right] .
$$

This generic form mimics a non-Vegard behavior, typical for layered intercalation compounds. ${ }^{17}$ As depicted in Fig. 4(a), the dilatation is weak at low densities $\left(x \ll x_{0}\right)$. The most intensive response is at $x \approx x_{0}$, and then the matrix reaches a saturation, corresponding to its mechanical stability limit. Here $\Delta$ is the matrix response constant or dilatation rate, controlling the slope near $x \approx x_{0}$. Such a threshold behavior of the volume variation with the guest concentration is well documented for various layered matrices ${ }^{3}$ (e.g., $\mathrm{Li}_{x} \mathrm{TiS}_{2}$ ). Therefore, accepting that the characteristic concentration $x_{0}$ increases with decreasing film thickness we can explain the observed crossover from thin to thick films. Growth of $x_{0}$ with decreasing thickness can be understood as a stronger influence of the film support, such that higher guest densities are required to induce detectable host dilatations. Having found a reasonable approximation for $p(x)$, we can solve Eq. (10) with respect to $\varphi(x)$. This function is plotted in Fig. 4(b). It is seen that, in agreement with our previous estimation, the distortion contribution changes its sign in the range of concentrations, close to the threshold point $x_{0}$. Therefore, in the low concentration region the distortion part $\varphi(x)$ for thick and thin films behaves differently. Namely, in the concentration domain where the volume dilatation is negligible $p(x) \approx 0$ or saturated $d p(x) / d x \approx 0$, the insertion is associated with an additional energy cost $\varphi(x)>0$ to distort the matrix. On the other hand, an increase in the volume allows for an optimization of the internal structure, and this makes the insertion locally more favorable $\varphi(x)<0$. Note that according 

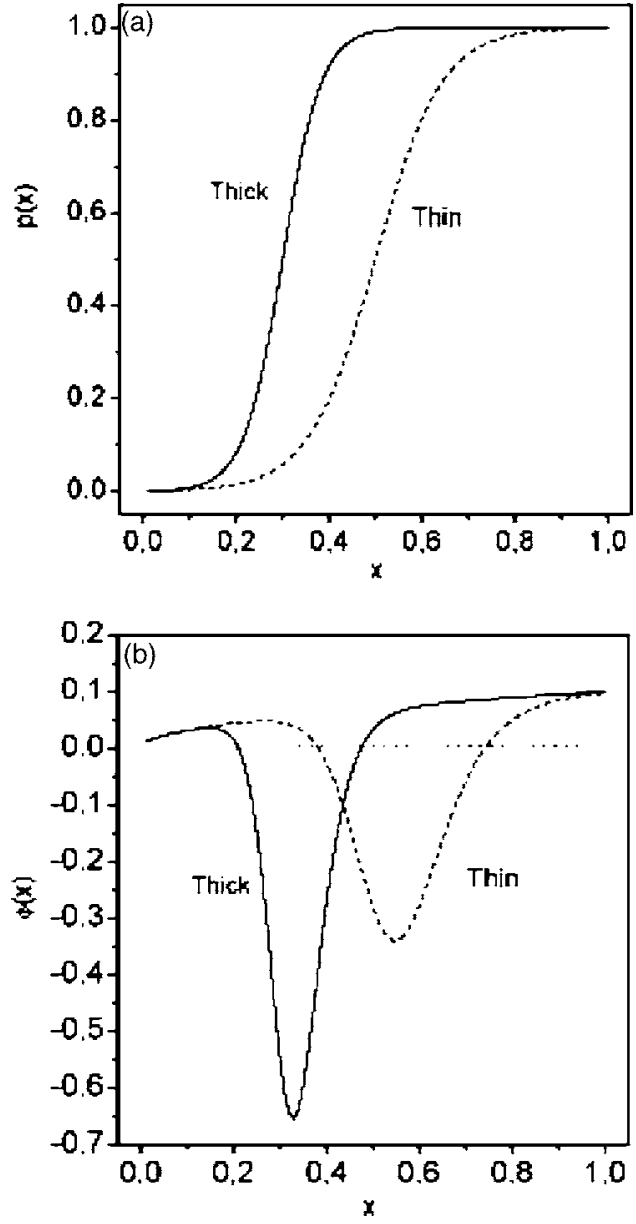

FIG. 4. (a) Modulating functions calculated from Eq. (16) at two characteristic concentrations $x_{0}=0.3$ (solid) and $x_{0}=0.5$ (dashed). (b) Corresponding distortion terms $\varphi(x)$ calculated from Eq. (10).

to Eq. (10) the sum of the dilatation and the distortion counterparts $(1+\eta) G x^{\eta}$ is always positive.

\section{CONCLUSION}

Thermodynamics of intercalation into disordered matrices is analyzed in terms of the maximum entropy principle combined with the distortive lattice gas model. This ap- proach allows us to account for the matrix volume expansion and the fluctuating internal distortions, seen in the recent experiments. ${ }^{13,14}$ Therefore, the crossover behavior from thin to thick films can be understood as a cooperative result of a thresholdlike host volume variation with the guest concentration $x$ and the internal distortions, optimizing the host-guest coupling. Our analysis points to the occurrence of the film support effect on the characteristic concentration $x_{0}$, corresponding to the volume dilatation onset. Decreasing thickness entails increasing $x_{0}$ values. A work on interpreting this tendency in terms of some physical film property is in progress. For instance, in the light of the present study, differences in the distorted amorphous structure (free volume) might be behind this effect. In order to verify this conjecture a systematic experimental study of the concentration dependent host morphology is desirable. This issue is left for a future study.

${ }^{1}$ L. Schlapbach and A. Zuttel, Nature (London) 414, 353 (2001).

${ }^{2}$ R. Matsuda, R. Kitaura, S. Kitagawa et al., Nature (London) 436, 238 (2005).

${ }^{3}$ W. R. McKinnon and R. R. Haering, in Modern Aspects of Electrochemistry, edited by R. E. White, J. O'M. Bockris, and B. E. Conway (Plenum, New York, 1983), Vol. 15, p. 235.

${ }^{4}$ C. M. Julien, Mater. Sci. Eng., R. 40, 47 (2003).

${ }^{5}$ L. D. Gelb, K. E. Gubbins, R. Radhakrishnan, and M. SliwinskaBartowiak, Rep. Prog. Phys. 62, 1573 (1999).

${ }^{6}$ T. Kudo and M. Hibino, Solid State Ionics 84, 65 (1996).

${ }^{7}$ M. Stromme Mattsson, Phys. Rev. B 58, 11015 (1998).

${ }^{8}$ F. Detcheverry, E. Kierlik, M. L. Rosinberg, and G. Tarjus, Phys. Rev. E 72, 051506 (2005).

${ }^{9}$ E. V. Vakarin and J. P. Badiali, Phys. Rev. E 74, 036120 (2006).

${ }^{10}$ F. A. Escobedo and J. J. de Pablo, J. Chem. Phys. 106, 793 (1997).

${ }^{11}$ G. Odriozola and J. F. Aguilar, J. Chem. Phys. 123, 174708 (2005).

${ }^{12}$ P. Thibault, J. J. Prejean, and L. Puech, Phys. Rev. B 52, 17491 (2005).

${ }^{13}$ G. Garcia-Belmonte, J. Garcia-Canadas, and J. Bisquert, J. Phys. Chem. B 110, 4514 (2006).

${ }^{14}$ G. Garcia-Belmonte, V. S. Vikhrenko, J. Garcia-Canadas, and J. Bisquert, Solid State Ionics 170, 123 (2004).

${ }^{15}$ E. V. Vakarin, Y. Duda, and J. P. Badiali, J. Chem. Phys. 124, 144515 (2006); J. Phys. Chem. B 111, 2540 (2007).

${ }^{16}$ E. V. Vakarin and J. P. Badiali, Electrochim. Acta 50, 1719 (2005).

${ }^{17}$ E. V. Vakarin and J. P. Badiali, J. Phys. Chem. B 106, 7721 (2002).

${ }^{18}$ E. T. Jaynes, Phys. Rev. 106, 620 (1957).

${ }^{19}$ E. V. Vakarin and J. P. Badiali, Surf. Sci. 513, 431 (2002).

${ }^{20}$ E. V. Vakarin and J. P. Badiali, Surf. Sci. 565, 279 (2004). 